\title{
PERFORMANCE SUMMARY OF THE HELICAL MAGNETS FOR RHIC*
}

\author{
E. Willen, M. Anerella, J. Escallier, G. Ganetis, A. Ghosh, R. Gupta, M. Harrison, A. Jain, W. \\ MacKay, A. Marone, J. Muratore, S. Plate, R. Thomas, P. Wanderer and KC Wu, BNL, Upton, NY \\ 11973, USA \\ M. Okamura, RIKEN, Japan
}

\section{Abstract}

A series of four Snake and eight Rotator superconducting helical magnet assemblies has been built and installed in RHIC to control the polarization of protons during acceleration and storage in that machine. Each of these assemblies consists of four $2.4 \mathrm{~m}$ long dipole magnets in each of which the field rotates through 360 degrees along the magnet's length. The magnets were made by winding one millimeter diameter superconducting 7-strand cable into slots milled into thick-walled aluminum tubes. The magnets produce 4 Tesla field at a current of 320 amperes and are quenchprotected with $0.050 \mathrm{ohm}$ resistors placed across the winding in each slot. A total of 48 of these $2.4 \mathrm{~m}$ magnets has been built, tested and installed. This paper summarizes their quench performance as well as their field uniformity, of which the integral field is the most critical. All magnets reached the required operating field level of $4 \mathrm{~T}$, and the integral field of the magnets was generally about half of the maximum permissible level of 0.050 Tesla meters.

\section{INTRODUCTION}

Magnets to control proton spin were required in RHIC to enable a program of spin physics using polarized proton collisions [1]. These magnets precess the proton spin from up to down and back again on each orbit around the ring, thereby avoiding depolarizing resonances during acceleration. They also precess the proton spin at each of two experimental detectors from vertical to longitudinal for the study of such oriented collisions. The magnets were built over several years and are now completely installed and are operational beginning with the 2003 RHIC running period.

\section{LAYOUT}

To achieve the required spin rotations, helical magnets were developed in which the dipole field rotates through $360^{\circ}$ in a distance of $2.4 \mathrm{~m}$. Helical magnets offered a more compact and efficient design than could be achieved with a combination of rotated dipole magnets. Precessing the proton spin without a net deflection of the orbit was achieved by combining four of the helical magnets into one long cryostatted device with different field strength, helix direction, and helix orientation in each device, depending on its task. They are called "Snakes" because of the serpentine particle trajectory through the device. The available space in the RHIC lattice determined the overall length and therefore the required field of the magnets. The coil inner diameter is a large $100 \mathrm{~mm}$ to *Work supported in part by the U.S. Department of Energy. allow adequate space for the beam trajectory (the RHIC dipoles have a coil aperture of $80 \mathrm{~mm}$ ). Four Snakes were required, two in each ring, to avoid depolarizing resonances, and eight Snakes, also called Rotators, were required to orient the spin at the detectors. Thus, a total of $48,2.4 \mathrm{~m}$ long helical magnets was required. A low operating current was needed to ease the cryogenic load from the numerous power leads to these magnets, which are spread around the ring.

\section{DESIGN}

The design has been described in previous papers [2-4]. Briefly, the coils are made by placing conductor, a Kapton-wrapped cable made of seven, twisted $0.33 \mathrm{~mm}$ $\mathrm{NbTi}$ superconducting wires, into slots milled into aluminum cylinders. These cylinders with their windings are later overwrapped with a wet layup of fiberglass/epoxy, then machined to a precise diameter. Each magnet has an inner coil with seven slots and an outer coil with nine slots. The two coils are assembled into a laminated yoke made of one piece laminations to make a single, $2.4 \mathrm{~m}$ long helical dipole. End plates are added and electrical connections including quench protection resistors across each winding are made. Four of these helical dipoles are assembled into a stainless steel shell to complete the helium enclosure and the support structure for the final cryostatted magnet, which operates with forced flow helium at $4.5 \mathrm{~K}$. A few parameters of the dipole magnet are given in Table I.

Table I: Selected parameters of the helical dipole.

\begin{tabular}{lcc}
\hline \multicolumn{1}{c}{ Parameter } & Units & Value \\
\hline Aperture & $\mathrm{mm}$ & 100 \\
Magnetic length & $\mathrm{m}$ & 2.4 \\
Field & $\mathrm{T}$ & 4 \\
Current & $\mathrm{A}$ & 320 \\
Number of turns & & 1680 \\
Inductance & $\mathrm{H}$ & 4.8 \\
Stored energy @ 4 T & $\mathrm{kJ}$ & 240 \\
Diameter of yoke & $\mathrm{mm}$ & 355.6 \\
Num. of strands in cable & & 7 \\
Strand diameter & $\mathrm{mm}$ & 0.33 \\
Cu to non-Cu ratio & & $2.5: 1$ \\
\hline
\end{tabular}

\section{CONSTRUCTION}

The coils are constructed by manually placing the Kapton-wrapped cable into the slots in an orderly array. The sides of the slots have been previously insulated with Kapton for good electrical insulation. A layer of prepreg 
material (B-stage epoxy/fiberglass cloth) is placed between each layer. When all the turns are in place, press plates are placed on top of the windings and the coil is wrapped with tensioned Kevlar cord, then placed in an oven where the epoxy is cured while the coil rotates. The turns move radially inward as the epoxy softens and flows in this curing operation. The end result is a compact matrix of cable turns, Kapton, fiberglass and epoxy filling the slot. A photograph of a coil is shown in Fig. 1.

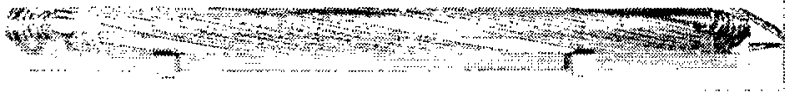

Figure 1: Photograph of a helical coil with conductors.

The thickness of the prepreg material, initially $0.25 \mathrm{~mm}$ but finally $0.075 \mathrm{~mm}$ after compression, at first keeps the turns at a larger radial position. As the turns are heated and moved radially inward by the tensioned Kevlar, extra cable length is generated because of the helix along the length and because of the crossover from side to side in the ends. This extra length is accommodated by providing greater slot width in the ends. The extra space, too large to be completely filled with the prepreg epoxy, is later filled with ceramic-loaded epoxy.

\section{MEASUREMENTS}

Numerous electrical tests during construction ensured that most construction faults were caught. An occasional turn-to-turn short or coil-to-ground fault occurred in the construction program caused by misplaced turns, generally from cable buckling while curing. Abbreviated warm measurements verified that all windings were connected in the proper sequence. All the 48 magnets were cryogenically tested for quench performance and field quality. Six magnets developed faults during or after cryogenic testing and required disassembly and rewinding of a particular winding in the coil. Detailed field measurements for each magnet are available in various formats at Brookhaven.

\section{Training}

Most of the 48 magnets required some training to reach the desired $4 \mathrm{~T}$ field $(320 \mathrm{~A})$. Fig. 2 shows the distribution of first quenches and Fig. 3 shows the number of quenches before the current exceeded $320 \mathrm{~A}$. Nine magnets required no training, 28 required three or less, and 11 required more than three. The training is attributed to a paucity of epoxy in the windings, revealed as voids in those windings that were on occasion taken apart or replaced to correct faults. This was a problem particularly in the ends where slots were wider. Several of the early coils required excessive training (up to 18 quenches) but this problem improved as experience in placing and compressing the cable into the slots was gained by the technical staff. Virtually all the training occurred in the inner coils in one of the windings away from the midplane, the region of highest Lorentz forces and therefore as expected. In those magnets with thermal cycles, the magnets for the most part remembered their training, except for several of the early magnets where some retraining was necessary. This is shown in Fig. 4.

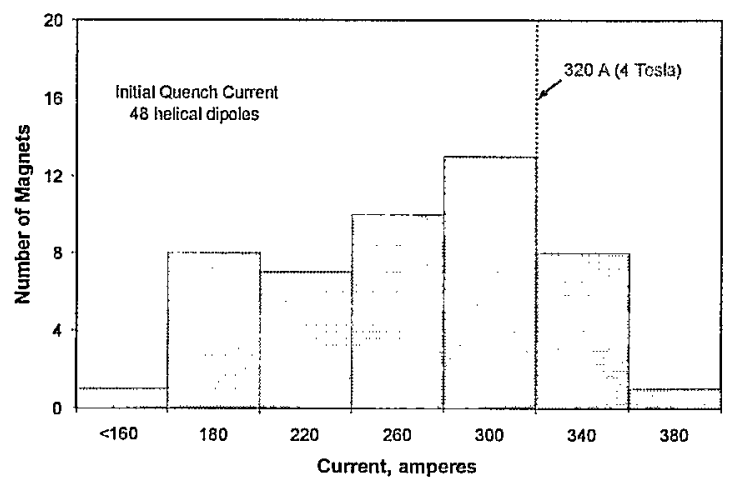

Figure 2: Distribution of initial quench currents.

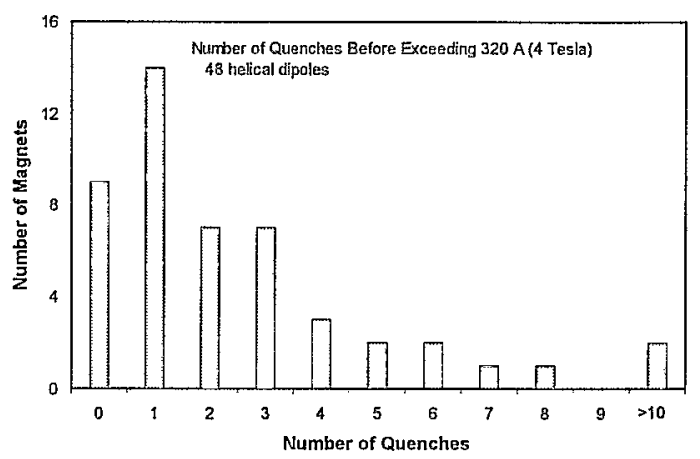

Figure 3: Number of required quenches.

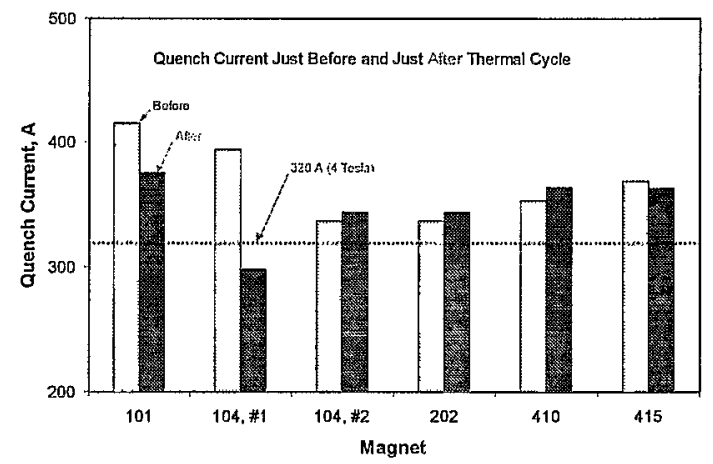

Figure 4: Retraining after thermal cycle.

\section{Integral Field}

An important requirement for the helical magnets was that the integral field be small or zero in order to avoid net deflections of the beam. The specification was that the field integral be less than $0.025 \mathrm{~T} \cdot \mathrm{m}$. This was measured with a long, rotating coil with a radius of $25 \mathrm{~mm}$ that extended the full length of the magnet including any fringe field at the ends. A typical measurement is shown in Fig. 5 where the maximum of the residual is $0.018 \mathrm{~T} \cdot \mathrm{m}$, well within the required limit. This was typical for all the magnets; none had a value greater than $50 \%$ of the limit. 


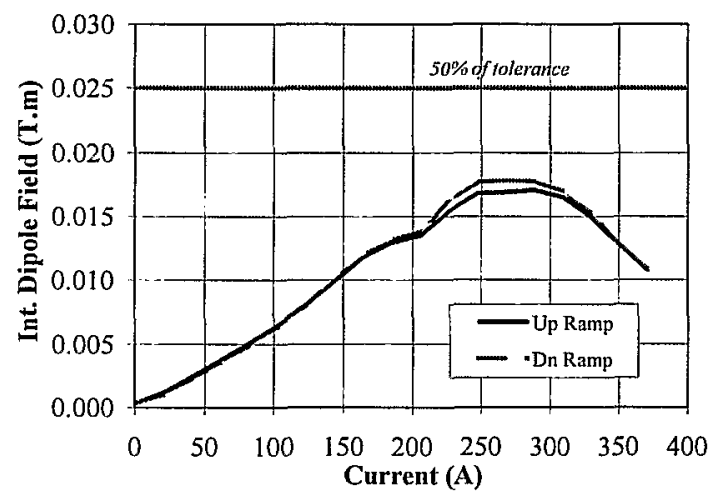

Figure 5: Example of measured integral field. The structure in the curves is due to limitations in the uniformity of the measuring coil.

\section{Multipoles}

In a helical magnet, all field multipoles (harmonics) oscillate with axial position. Over the length of the magnet, the effect of these multipoles on the particle trajectory tends to cancel, so the requirements on field quality are less stringent than for normal accelerator magnets: the local $\Delta|\mathrm{B}| /|\mathrm{B}|$ should be kept reasonably below $1 \%$. A rotating coil to measure the harmonics should be as short as possible in order to avoid signal cancellation. The measuring coil used to measure these magnets was only $51 \mathrm{~mm}$ long, about $2 \%$ of the wavelength of the helix. It had a radius of $34.2 \mathrm{~mm}$, and the reference radius used to express the harmonics is 31 $\mathrm{mm}$. A total of 56 turns in the tangential winding of the coil ensured adequate signal strength for good measurements. Flux cancellation because of the helical field reduces the dipole field by $0.25 \%$ for the dipole term and by $2.7 \%$ for the 30 -pole term, an effect corrected in the reported results.

Measurements at many currents were typically made at a single location in the center of the magnet and as a function of axial position at several currents [5]. The axial variations of the quadrupole and the sextupole components are shown in Fig. 6. The asymmetry in the quadrupole component along the length of the magnet varies magnet to magnet but the data shown are typical and are believed to result from a combination of coil asymmetries inside the iron yoke and the centering technique used. The variation of the sextupole amplitude with current results from iron saturation in the yoke.

The normal sextupole field as a function of current is shown in Fig. 7 (left) for most of the magnets on a single plot. The right side shows the same data normalized to zero at $102 \mathrm{~A}$ on the up ramp. The harmonics are expressed in a coordinate system where the $y$-axis coincides with the dipole field direction. This allowed harmonic is quite consistent over all the magnets.

The following conclusions can be drawn:

- There is considerable magnet-to-magnet variation in the harmonics resulting from geometric differences in the magnets.
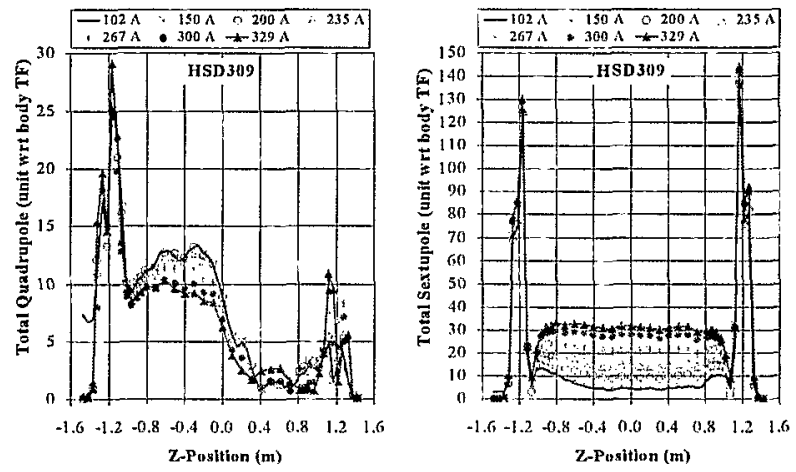

Figure 6: Example of measured quadrupole and sextupole fields in a typical magnet.
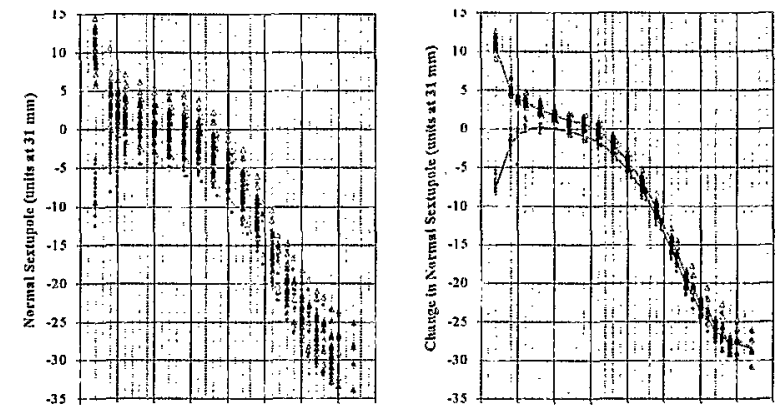

Figure 7: Unnormalized (left) and normalized sextupole harmonic for the magnets.

- The current dependence of the harmonics has small magnet-to-magnet variations.

- Z-scans in small axial increments show significant axial variation in the lower order harmonics.

- All harmonics are within safe limits for machine operation.

\section{REFERENCES}

Note: A number of these papers are available at the BNL web site.

[1] Design Manual: Polarized Proton Collider at RHIC, M. Syphers, Editor, BNL, July, 1998.

[2] E. Willen, R. Gupta, A. Jain, E. Kelly, G. Morgan, J. Muratore, R. Thomas, A Helical Magnet Design for RHIC, PAC97, Vancouver, May, 1997.

[3] E. Willen, E. Kelly, M. Anerella, J. Escallier, G. Ganetis, A. Ghosh, A. Jain, A. Marone, G. Morgan, J. Muratore, A. Prodell, P. Wanderer, Construction of Helical Magnets for RHIC, PAC99, New York, March/April, 1999.

[4] E. Willen, M. Anerella, J. Escallier, G. Ganetis, A. Ghosh, A. Jain, E. Kelly, G. Morgan, J. Muratore, A. Prodell, P. Wanderer, Performance of Helical Magnets for RHIC, MT16, Ponte Vedra, FL, September/October 1999.

[5] A. Jain, G. Ganetis, W. Louie, A. Marone, J, Muratore, R. Thomas, P. Wanderer, E. Willen, Measurements of Field Quality in Helical Dipoles for RHIC, Proc. 12th International Magnet Measurement Workshop, October, 2002. 